\title{
A population-based cohort study of mortality of intensive care unit patients with liver cirrhosis
}

\author{
Yu-Feng Huang 1,2, Chao-Shun Lin ${ }^{3,4,5}$, Yih-Giun Cherng ${ }^{2,3}$, Chun-Chieh Yeh ${ }^{6,7}$, Ray-Jade Chen ${ }^{8,9}$, \\ Ta-Liang Chen ${ }^{3,10}$ and Chien-Chang Liao $3,4,5,11,12^{*}$ (D)
}

\begin{abstract}
Background: The impact of liver cirrhosis on the outcomes of admission to intensive care unit (ICU) is not completely understood. Our purpose is to identify risk factors for mortality in ICU patients with liver cirrhosis.

Methods: Using reimbursement claims from Taiwan's National Health Insurance Research Database from in 20062012, 1,250,300 patients were identified as having ICU stays of more than 1 day, and 37,197 of these had liver cirrhosis. With propensity score-matching for socioeconomic status, pre-existing medical conditions, and cirrhosisrelated morbidities, 37,197 ICU patients without liver cirrhosis were selected for comparison. Adjusted odds ratios (ORs) and 95\% confidence intervals (Cls) of cirrhosis associated with 30-day, ICU, and one-year mortality were calculated.

Results: Compared with control, cirrhotic patients had higher 30-day mortality (aOR 1.60, 95\% Cl 1.53 to 1.68), particularly those with jaundice ( $\mathrm{aOR} 2.23,95 \% \mathrm{Cl} 2.03$ to 2.45), ascites (aOR 2.32, 95\% Cl 2.19 to 2.46) or hepatic coma (aOR 2.21, 95\% Cl 2.07 to 2.36). Among ICU patients, liver cirrhosis was also associated with ICU mortality (aOR $144,95 \% \mathrm{Cl} 1.38$ to 1.51 ) and one-year mortality (aOR 1.40, 95\% Cl 1.35 to 1.46). Associations between cirrhosis of liver and increased 30-day mortality were significant in both sexes and every age group.

Conclusions: Liver cirrhosis was associated with 30-day mortality in ICU patients. Jaundice, ascites, hepatic coma, more than 4 admissions due to cirrhosis, and more than 30 days of hospital stay due to cirrhosis were exacerbated factors in cirrhotic ICU patients.
\end{abstract}

Keywords: Intensive care unit, Liver cirrhosis, Mortality

\section{Background}

Cirrhosis of the liver results in various complications and mortality worldwide, but especially in developed regions [1]. It is the fourth most common cause of death in Europe and leads to more than a million deaths around the globe annually [2,3]. The main etiologies of liver cirrhosis in most areas are infection with hepatitis $\mathrm{B}$ or $\mathrm{C}$ virus and alcohol abuse [1]. A French screening program estimated prevalence at $0.3 \%$, and European

\footnotetext{
* Correspondence: jacky48863027@yahoo.com.tw; ccliao@tmu.edu.tw ${ }^{3}$ Department of Anesthesiology, School of Medicine, College of Medicine, Taipei Medical University, Taipei, Taiwan

${ }^{4}$ Department of Anesthesiology, Taipei Medical University Hospital, Taipei, Taiwan

Full list of author information is available at the end of the article
}

studies found annual incidences of $15.3-132.6$ per 100 , 000 people [3].

In the United States, annual medical expenditures related to intensive care unit (ICU) admissions are US \$3 billion, with mean charges of US $\$ 116,200$ per admission [4]. Patients in late-stage liver cirrhosis are likely to be admitted to ICUs for critical conditions such as sepsis and renal or respiratory failure $[4,5]$. Though some studies reported improving outcomes in patients with cirrhosis admitted to ICUs [6], the prognosis remains poor, with mortality rates as high as $45 \%$ or even higher $[7,8]$.

It is important to identify the risk factors of cirrhosis of the liver and associated adverse outcomes of patients admitted to ICU. Several prognostic scoring systems

(c) The Author(s). 2020 Open Access This article is distributed under the terms of the Creative Commons Attribution 4.0 International License (http://creativecommons.org/licenses/by/4.0/), which permits unrestricted use, distribution, and reproduction in any medium, provided you give appropriate credit to the original author(s) and the source, provide a link to the Creative Commons license, and indicate if changes were made. The Creative Commons Public Domain Dedication waiver (http://creativecommons.org/publicdomain/zero/1.0/) applies to the data made available in this article, unless otherwise stated. 
have been proposed for risk assessment [9-12], such as the Child-Pugh score [13], the Model for End-stage Liver Disease [14], the Acute Physiology and Chronic Health Evaluation [15], and the Sequential Organ Failure Assessment [16]. Most previous reports only analyzed risk factors that predict outcomes when stratifying cirrhotic patients admitted to ICU, but did not assess the influence of cirrhosis itself on mortality in ICU or one-year survival after discharge.

We conducted a nationwide population-based retrospective cohort study using Taiwan's National Health Insurance Research Database to investigate ICU mortality in patients with and without liver cirrhosis. We also evaluated the impacts of different comorbidities and cirrhosis-related clinical indictors on ICU mortality and on one-year survival in further stratified analyses.

\section{Methods}

\section{Data sources}

We conducted this study using reimbursement claims data from Taiwan's National Health Insurance Program. This program merged former insurance systems in March 1995 and covers more than 99\% of Taiwan's 23 million residents. The National Health Research Institutes established a National Health Insurance Research Database (NHIRD) to record all beneficiaries' inpatient and outpatient medical services. This information includes basic patient demographics, physician's primary and secondary disease diagnoses, treatment procedures, prescribed medications and medical expenditures for all health care services. The validity of this database has been favorably evaluated, and research articles based on it have been accepted in prominent scientific journals worldwide [17-20].

\section{Ethics}

This study was conducted in accordance with the Helsinki Declaration. To protect personal privacy, the electronic database was decoded with patient identifications scrambled for further academic access for research. According to Taiwan National Health Research Institutes regulations, informed consent is not required because patient identifications were decoded and scrambled [18-20]. Ethical approval for this study (TMU-JIRB-201504008) was provided by the Institutional Review Board of Taipei Medical University.

\section{Study design}

Among 23 million beneficiaries, 1,250,300 patients were admitted to ICU between 2006 and 2012 (Additional file 1: Figure S1). We identified 79,528 patients aged $\geq 20$ years who had histories of liver cirrhosis from the National Health Insurance Research Database. Patients with liver cirrhosis were defined as having at least two visits for medical care with physician's primary diagnosis of liver cirrhosis within the 24 months before ICU admission. To select appropriate comparison groups, we matched each ICU patient with cirrhosis with one randomly selected ICU patients without liver cirrhosis by the analysis with a propensity score-matched pair procedure (case-control ratio $=1: 1$ ). These matched factors included age, sex, low income, stay in medical center or not, diabetes, hypertension, mental disorder, chronic obstructive pulmonary disease, fracture, pneumonia, stroke, asthma, traumatic brain injury, congestive heart failure, immune thrombocytopenia, renal dialysis, hyperlipidemia, epilepsy, atrial fibrillation, peripheral vascular disease and systemic lupus erythematosus, causes of admission to ICU according to physician's primary diagnosis (digestive disease, cancer, respiratory disease, circulatory disease, infectious disease, injury and poisoning, symptom-defined conditions, genitourinary disease, endocrine disease, musculoskeletal disease, neurological disease, skin disease, mental disorder, tumors, blood diseases, congenital anomalies, disease of perinatal period, complications of pregnancy, ICU complications (such as septicemia, pneumonia, acute renal failure, urinary tract infection, stroke, acute myocardial infarction and pulmonary embolism). After matching selection, there were 37,197 patients with cirrhosis of liver in the exposure group and 37,197 people without liver cirrhosis in nonexposure group. We investigated the impact of liver cirrhosis on 30-day mortality, ICU mortality, and one-year mortality among ICU patients in this study.

\section{Measures and definitions}

Income status was identified by defining low-income patients as those who qualified for waived medical copayment, as this status is verified by the National Health Insurance Bureau. Whether patients stayed in medical center ICUs or those in other hospitals was also recorded. We used the International Classification of Diseases, Ninth Revision, Clinical Modification (ICD-9-CM) to define coexisting medical conditions and ICU complications. Details codes of ICD-9-CM for these diseases were listed in Additional file 1: Table S1. Cirrhosis of liver before ICU stay was defined as the major exposure. Coexisting medical conditions determined from medical claims within the 24-month period before ICU stay included diabetes, hypertension, mental disorders, chronic obstructive pulmonary disease, fracture, pneumonia, stroke, asthma, traumatic brain injury, congestive heart failure, immune thrombocytopenia, hyperlipidemia, epilepsy, atrial fibrillation, peripheral vascular disease, and systemic lupus erythematosus. Renal dialysis was defined by administration code (D8, D9). Seven major complications during the ICU stay were analyzed (and those having severe cases of these diseases before ICU were 
excluded) including septicemia, pneumonia, acute renal failure, urinary tract infection, stroke, acute myocardial infarction, and pulmonary embolism. Length of hospital stay and ICU medical expenditure were analyzed as secondary outcomes.

Causes of admission to ICU (according to physician's primary diagnosis at admission) were also identified and described with disease codes including digestive disease, cancer, respiratory disease, circulatory disease, infectious disease, injury and poisoning, symptom-defined conditions, genitourinary disease, endocrine disease, musculoskeletal disease, neurological disease, skin disease, mental disorder, tumor, blood disease, congenital anomalies, disease of perinatal period, and pregnancy complications.

\section{Statistical analysis}

To reduce confounding bias, we used a propensity score-matched pair combined with frequency matching procedure to balance the covariates between ICU patients with and without liver cirrhosis. We developed a non-parsimonious multivariable logistic regression model to estimate a propensity score for pre-ICU cirrhosis of the liver. We matched cirrhotic patients to patients without liver cirrhosis, using a greedy matching algorithm (without replacement) with a caliper width of 0.2 SDs of the log odds of the estimated propensity score. Clinical significance guided initial choices of covariates in this multivariable logistic regression model: age, sex, low income, ICU stay in medical center or not, diabetes, hypertension, mental disorders, chronic obstructive pulmonary disease, fracture, pneumonia, stroke, asthma, traumatic brain injury, congestive heart failure, immune thrombocytopenia, renal dialysis, hyperlipidemia, epilepsy, atrial fibrillation, peripheral vascular disease, systemic lupus erythematosus, septicemia, pneumonia, acute renal failure, urinary tract infection, stroke, acute myocardial infarction, pulmonary embolism, digestive disease, cancer, respiratory disease, circulatory disease, infectious disease, injury and poisoning, symptomdefined conditions, genitourinary disease, endocrine disease, musculoskeletal disease, neurological disease, skin disease, mental disorder, tumor, blood disease, congenital anomalies, disease of perinatal period, and complications of pregnancy. A structured iterative approach was used to refine this model to achieve covariate balance within matched pairs. We used chi-square tests to measure covariate balance, and $p<0.05$ was suggested to represent meaningful covariate imbalance. We matched patients with and without cirrhosis using a greedymatching algorithm with a caliper width of $0.2 \mathrm{SD}$ of the $\log$ odds of the estimated propensity score. This method could remove $98 \%$ of bias from measured covariates.

Adjusted odds ratios (aORs) with 95\% confidence intervals (CIs) for 30-day mortality, ICU mortality, and one-year mortality for patients with and without cirrhosis were analyzed with multiple logistic regression models by controlling for age, sex, low income, stay in medical center or not, coexisting medical conditions, ICU complications and admission causes. To confirm associations between liver cirrhosis and ICU mortality, we also performed stratification analysis by age, sex, low income, stay in medical center or not, coexisting medical conditions, ICU complications and causes of ICU admission. The impacts of liver-related indicators and medical care on 30-day mortality in ICU patients with cirrhosis were also measured by calculating adjusted ORs and 95\% CIs in the multivariate logistic regression models. SAS version 9.1 (SAS Institute Inc., Cary, NC, USA) statistical software was used for data analyses; two-sided $p<$ 0.05 indicated significant differences.

\section{Results}

The Additional file 1: Figure S1 shows the flow chart for selecting ICU patients with and without liver cirrhosis. Table 1 shows the distributions of age, sex, low income, stay in medical center or not, coexisting medical conditions (diabetes, hypertension, mental disorders, chronic obstructive pulmonary disease, fracture, pneumonia, stroke, asthma, traumatic brain injury, congestive heart failure, idiopathic thrombocytopenic purpura, renal dialysis, hyperlipidemia, epilepsy, atrial fibrillation, peripheral vascular disease, systemic lupus erythematosus), ICU complications (septicemia, pneumonia, acute renal failure, urinary tract infection, stroke, acute myocardial infarction, pulmonary embolism), causes of admission to ICU (digestive disease, cancer, respiratory disease, circulatory disease, infectious disease, injury and poisoning, symptom-defined conditions, genitourinary disease, endocrine disease, musculoskeletal disease, neurological disease, skin disease, mental disorders, tumor, blood disease, congenital anomalies, disease of perinatal period, pregnancy complications) as well as surgery and endotracheal intubation balanced between surgical patients with and without cirrhosis of liver using the matching procedure by propensity score.

In Table 2, patients with cirrhosis showed higher ICU medical expenditure than patients without $(12,008 \pm 9890$ vs. $11,366 \pm 8742$ USD, $p<0.0001$ ). Cirrhosis was associated with a significant increase in 30-day mortality (aOR $1.60,95 \%$ CI 1.53 to 1.68 ), ICU mortality (aOR 1.44 , $95 \%$ CI 1.38 to 1.51 ), and one-year mortality (aOR 1.40, 95\% CI 1.35 to 1.46) in ICU patients.

Compared with ICU patients without cirrhosis (Table 3), cirrhotic ICU patients had increased 30-day mortality when they also had liver cancer (aOR 1.85, 95\% CI 1.74 to 1.97), hepatitis $\mathrm{B}$ or $\mathrm{C}$ virus infection (aOR 1.75, 95\% CI 1.50 to 2.03), alcohol dependence syndrome (aOR 1.82, 95\% CI 1.62 to 2.04), jaundice (aOR 2.23, 95\% CI 2.03 to 
Table 1 Characteristics of ICU patients with and without liver cirrhosis

\begin{tabular}{|c|c|c|c|c|}
\hline \multirow{2}{*}{$\begin{array}{l}\text { Pre-hospital and in-hospital characteristics } \\
\text { Age, years }\end{array}$} & \multicolumn{2}{|c|}{$\begin{array}{l}\text { No liver cirrhosis } \\
(N=37197)\end{array}$} & \multicolumn{2}{|c|}{$\begin{array}{l}\text { Liver cirrhosis } \\
(N=37197)\end{array}$} \\
\hline & $\mathrm{n}$ & (\%) & $\mathrm{n}$ & (\%) \\
\hline $20-29$ & 304 & $(0.8)$ & 304 & $(0.8)$ \\
\hline 30-39 & 2261 & (6.1) & 2261 & (6.1) \\
\hline $40-49$ & 6022 & (16.2) & 6022 & (16.2) \\
\hline 50-59 & 8939 & (24.0) & 8939 & (24.0) \\
\hline 60-69 & 7832 & (21.1) & 7832 & (21.1) \\
\hline 70-79 & 7841 & (21.1) & 7841 & (21.1) \\
\hline$\geq 80$ & 3998 & (10.8) & 3998 & $(10.8)$ \\
\hline \multicolumn{5}{|l|}{ Sex } \\
\hline Female & 10,274 & (27.6) & 10,274 & (27.6) \\
\hline Male & 26,923 & (72.4) & 26,923 & (72.4) \\
\hline Low income & 1192 & (3.2) & 1192 & (3.2) \\
\hline ICU in medical center & 11,331 & (30.5) & 11,331 & (30.5) \\
\hline \multicolumn{5}{|l|}{ Coexisting medical conditions } \\
\hline Hypertension & 8949 & (24.1) & 8949 & (24.1) \\
\hline Diabetes & 8360 & (22.5) & 8360 & (22.5) \\
\hline Mental disorder & 6725 & (18.1) & 6725 & (18.1) \\
\hline Peptic ulcer disease & 6206 & (16.7) & 6206 & (16.7) \\
\hline Anemia & 4507 & (12.1) & 4507 & (12.1) \\
\hline COPD & 3561 & (9.6) & 3561 & (9.6) \\
\hline Fracture & 2495 & (6.7) & 2495 & (6.7) \\
\hline Asthma & 1250 & (3.4) & 1250 & (3.4) \\
\hline Atherosclerosis & 1197 & (3.2) & 1197 & (3.2) \\
\hline Traumatic brain injury & 1063 & (2.9) & 1063 & (2.9) \\
\hline Congestive heart failure & 832 & (2.2) & 832 & (2.2) \\
\hline Renal dialysis & 806 & (2.2) & 806 & (2.2) \\
\hline Thrombocytopenia & 719 & (1.9) & 719 & (1.9) \\
\hline \multicolumn{5}{|l|}{ Reasons for ICU admission } \\
\hline Digestive disease & 10,198 & (27.4) & 10,198 & (27.4) \\
\hline Cancer & 6726 & (18.1) & 6726 & (18.1) \\
\hline Respiratory disease & 4524 & (12.2) & 4524 & (12.2) \\
\hline Circulatory disease & 4385 & (11.8) & 4385 & (11.8) \\
\hline Infectious disease & 3518 & $(9.5)$ & 3518 & (9.5) \\
\hline Injury and poisoning & 2918 & (7.8) & 2918 & (7.8) \\
\hline Symptom-defined conditions & 862 & (2.3) & 862 & (2.3) \\
\hline Genitourinary disease & 762 & (2.0) & 762 & (2.1) \\
\hline Endocrine disease & 605 & (1.6) & 605 & (1.6) \\
\hline Musculoskeletal disease & 598 & (1.6) & 598 & (1.6) \\
\hline Mental disorder & 278 & $(0.8)$ & 278 & $(0.8)$ \\
\hline Neurological disease & 304 & $(0.8)$ & 304 & $(0.8)$ \\
\hline Skin diseases & 291 & $(0.8)$ & 291 & $(0.8)$ \\
\hline Tumor & 254 & $(0.7)$ & 254 & $(0.7)$ \\
\hline Blood disease & 76 & $(0.2)$ & 76 & $(0.2)$ \\
\hline Congenital anomalies & 40 & $(0.1)$ & 40 & $(0.1)$ \\
\hline Disease of perinatal period & 33 & $(0.1)$ & 33 & $(0.1)$ \\
\hline Pregnancy complications & 7 & $(0.02)$ & 7 & $(0.02)$ \\
\hline Receiving surgery & 15,297 & (41.1) & 15,297 & (41.1) \\
\hline Endotracheal intubation & 13,076 & (35.1) & 13,076 & (35.1) \\
\hline \multicolumn{5}{|l|}{ Complications in ICU } \\
\hline Septicemia & 7338 & (19.7) & 7338 & (19.7) \\
\hline Pneumonia & 2970 & (8.0) & 2970 & (8.0) \\
\hline Acute renal failure & 1708 & (4.6) & 1708 & (4.6) \\
\hline Urinary tract infection & 1681 & $(4.5)$ & 1681 & $(4.5)$ \\
\hline Stroke & 1438 & (3.9) & 1438 & (3.9) \\
\hline Acute myocardial infarction & 403 & $(1.1)$ & 403 & $(1.1)$ \\
\hline Pulmonary embolism & 22 & $(0.1)$ & 22 & $(0.1)$ \\
\hline
\end{tabular}


Table $\mathbf{2}$ Intensive care unit mortality in patients with and without liver cirrhosis

\begin{tabular}{lllll}
\hline Outcomes of ICU & No LC, \% & LC, \% & OR & $(95 \%$ CI) \\
\hline 30-day mortality & 10.9 & 15.9 & 1.60 & $(1.53$ to 1.68$)$ \\
ICU mortality & 12.9 & 17.2 & 1.44 & $(1.38$ to 1.51$)$ \\
One-year mortality & 17.3 & 22.1 & 1.40 & $(1.35$ to 1.46$)$ \\
Medical expenditure, USD & & \\
Length of hospital stay, days $^{\mathrm{b}}$ & $11,366 \pm 8742$ & $12,008 \pm 0.0001$ & $p=0.2174$ \\
\hline
\end{tabular}

$\mathrm{Cl}$ confidence interval, ICU intensive care unit, $O R$ odds ratio

${ }^{a}$ Adjusted all.covariates listed in Table 1

${ }^{\mathrm{b}}$ Mean \pm SD

2.45), ascites (aOR 2.32, 95\% CI 2.19 to 2.46), gastrointestinal hemorrhage (aOR 1.90, 95\% CI 1.78 to 2.03), hepatic coma (aOR 2.21, 95\% CI 2.07 to 2.36), more than 4 admissions due to LC (aOR 2.52, 95\% CI 2.20 to 2.89), more than 30 days of hospital stay due to LC (aOR 2.97, 95\% CI 2.66 to 3.31 ), and albumin supplement (aOR 1.93, 95\% CI 1.83 to 2.04). The aORs of alcohol-related cirrhosis and previous hospitalization associated with 30-day mortality were 1.75 (95\% CI 1.66 to 1.85 ) and 1.52 (95\% CI 1.46 to 1.58), respectively.

The association between liver cirrhosis and ICU mortality was significant in relation to the following causes of ICU admission: digestive disease (aOR 3.23, 95\% CI 2.91 to 3.58 ), cancer (aOR $1.15,95 \%$ CI 1.05 to 1.27 ), respiratory disease (aOR 1.24, 95\% CI 1.12 to 1.38 ), circulatory disease (aOR 1.47, 95\% CI 1.28 to 1.70 ), infectious disease (aOR 1.32, 95\% CI 1.19 to 1.48 ), injury and poisoning (aOR 1.95, 95\% CI 1.61 to 2.35), symptomdefined conditions (aOR 1.86, 95\% CI 1.43 to 2.42), genitourinary disease (aOR 1.74, 95\% CI 1.10 to 2.75 ), musculoskeletal disease (aOR 3.49, 95\% CI 1.79 to 6.82), and neurological disease (aOR 2.94, 95\% CI 1.30 to 6.65). The 30-day mortality was also associated with LC in ICU patients with 2 scores (aOR 1.49, 95\% CI 1.31 to 1.70), 3 scores (aOR 1.45, 95\% CI 1.25 to 1.69), and 4 scores (aOR 1.40, 95\% CI 1.17 to 1.67 ) of Charlson Comorbidity Index.

Stratified analysis and effects of cirrhosis-related clinical indicators on ICU mortality and one-year mortality of ICU patients were showed in Additional file 1: Tables S2 and S3. The actual survival starting at the day of ICU admission in patients with and without liver cirrhosis was showed in Additional file 1: Table S4.

\section{Discussion}

In this large-scale, nationwide, population-based propensity score-matched study, patients with cirrhosis of the liver admitted to ICU showed significantly higher ICU mortality as well as increased medical expenditure compared with non-cirrhotic controls. The stratified analyses showed higher ICU mortality among patients with increasing numbers of co-morbidities. Associated with even higher ICU mortality were cirrhosis-related clinical conditions, liver cancer, alcohol dependence syndrome, jaundice, ascites, gastrointestinal hemorrhage and hepatic coma. Regarding long-term outcomes after ICU discharge, higher one-year mortality was noted in liver cirrhosis patients with older age, male gender, ICU in medical center, anemia, renal dialysis, congestive heart failure, and complications in ICU such as septicemia and pneumonia.

To the best of our knowledge, this is the first report investigating the influence of liver cirrhosis on 30-day mortality, ICU mortality, and one-year mortality in ICU patients using a nationwide database. Previous studies were mostly conducted from a single center $[10,12,21-$ $24]$, which may represent a certain type of patient group and medical practice. Although these studies evaluated risk factors among patients with liver cirrhosis admitted to ICU, they did not note various cirrhosis-related clinical characteristics nor assess the impact of cirrhosis per se, adjusting all covariates with the control group to the ICU mortality [7, 10-12, 25, 26]. In our subgroup analyses, the odds ratios of ICU mortality in cirrhotic patients' ICU admissions with primary diagnoses such as cancer, respiratory disease and infectious disease were lower than other causes of ICU admission. This might be attributed to cancer, pneumonia, COPD and sepsis having more impact on ICU mortality than cirrhosis after adjustment [27-29].

Regarding the effects of cirrhosis-related clinical indicators on ICU mortality, cirrhotic patients with liver cancer, alcohol dependence syndrome, jaundice, ascites, gastrointestinal hemorrhage and hepatic coma had higher ICU mortality than those without cirrhosis. Of these cirrhosis-related clinical indicators, jaundice, ascites and hepatic coma were consistent with the ChildPugh score risk factors of the most commonly used prognosis predicting model in cirrhotic patients [13]. The development of ascites, gastrointestinal hemorrhage, encephalopathy and jaundice mark the decompensated stage of liver cirrhosis that results in poorer prognoses $[30,31]$. These complications might contribute to the increased ICU mortality found in patients of liver cirrhosis with more hospitalization. Liver cancer, especially hepatocellular carcinoma, is one of the leading causes of 
Table 3 Stratified analysis and effects of cirrhosis-related clinical indicators on 30-day mortality of ICU patients

\begin{tabular}{|c|c|c|c|c|c|}
\hline & \multirow[t]{2}{*}{$\mathrm{n}$} & \multicolumn{4}{|c|}{ 30-day mortality } \\
\hline & & Deaths & Mortality, \% & OR & $(95 \% C l)^{a}$ \\
\hline \multicolumn{6}{|l|}{ Pre-ICU characteristics within 2 years } \\
\hline No LC & 37,197 & 4046 & 10.9 & 1.00 & (reference) \\
\hline LC without liver cancer & 25,758 & 3705 & 14.4 & 1.48 & (1.41 to 1.56$)$ \\
\hline LC with liver cancer & 11,439 & 2190 & 19.2 & 1.85 & (1.74 to 1.97$)$ \\
\hline LC with no HBV and HCV & 22,258 & 3366 & 15.1 & 1.55 & (1.47 to 1.63$)$ \\
\hline LC with HBV or HCV & 13,573 & 2288 & 16.9 & 1.67 & (1.58 to 1.77$)$ \\
\hline LC with HBV and HCV & 1366 & 241 & 17.6 & 1.75 & (1.50 to 2.03$)$ \\
\hline LC without ADS & 34,496 & 5450 & 15.8 & 1.59 & (1.52 to 1.66$)$ \\
\hline LC with ADS & 2701 & 445 & 16.5 & 1.82 & (1.62 to 2.04$)$ \\
\hline LC without jaundice & 33,978 & 5171 & 15.2 & 1.54 & (1.47 to 1.62$)$ \\
\hline LC with jaundice & 3219 & 724 & 22.5 & 2.23 & (2.03 to 2.45$)$ \\
\hline LC without ascites & 26,352 & 3332 & 12.6 & 1.30 & (1.23 to 1.37$)$ \\
\hline LC with ascites & 10,845 & 2563 & 23.6 & 2.32 & (2.19 to 2.46$)$ \\
\hline LC without GI hemorrhage & 27,652 & 4114 & 14.9 & 1.50 & (1.43 to 1.58$)$ \\
\hline LC with Gl hemorrhage & 9545 & 1781 & 18.7 & 1.90 & (1.78 to 2.03 ) \\
\hline LC without hepatic coma & 29,252 & 4102 & 14.0 & 1.43 & (1.37 to 1.37$)$ \\
\hline LC with hepatic coma & 7945 & 1793 & 22.6 & 2.21 & (2.07 to 2.36$)$ \\
\hline LC with 0 admission & 25,677 & 3378 & 13.2 & 1.33 & (1.26 to 1.40$)$ \\
\hline LC with 1 admission & 6600 & 1411 & 21.4 & 2.16 & (2.01 to 2.32) \\
\hline LC with 2 admissions & 2449 & 521 & 21.3 & 2.14 & (1.92 to 2.38$)$ \\
\hline LC with 3 admissions & 1059 & 248 & 23.4 & 2.37 & (2.03 to 2.76 ) \\
\hline LC with $\geq 4$ admissions & 1412 & 337 & 23.9 & 2.52 & (2.20 to 2.89 ) \\
\hline LC with 0 days of hospital stay & 25,677 & 3378 & 13.2 & 1.33 & (1.26 to 1.40$)$ \\
\hline LC with 1-9 days of hospital stay & 5144 & 996 & 19.4 & 1.92 & (1.77 to 2.08$)$ \\
\hline LC with 10-19 days of hospital stay & 3022 & 664 & 22.0 & 2.23 & (2.02 to 2.45 ) \\
\hline LC with 20-29 days of hospital stay & 1312 & 305 & 23.3 & 2.30 & (2.00 to 2.65 ) \\
\hline LC with $\geq 30$ days of hospital stay & 2042 & 552 & 27.0 & 2.97 & (2.66 to 3.31$)$ \\
\hline LC without albumin supplement & 23,279 & 3140 & 13.5 & 1.40 & (1.33 to 1.47$)$ \\
\hline LC with albumin supplement & 13,918 & 2755 & 19.8 & 1.93 & (1.83 to 2.04$)$ \\
\hline LC without alcohol-related illness & 24,894 & 3581 & 14.4 & 1.25 & (1.20 to 1.31$)$ \\
\hline LC with alcohol-related illness & 12,303 & 2314 & 18.8 & 1.75 & (1.66 to 1.85$)$ \\
\hline LC without hospitalizations & 7214 & 756 & 10.5 & 0.91 & (0.84 to 0.98$)$ \\
\hline LC with hospitalizations & 29,983 & 5139 & 17.1 & 1.52 & (1.46 to 1.58$)$ \\
\hline \multicolumn{6}{|l|}{ Reasons for ICU admission } \\
\hline Digestive disease, no LC & 10,198 & 550 & 5.4 & 1.00 & (reference) \\
\hline Digestive disease, LC & 10,198 & 1511 & 14.8 & 3.23 & (2.91 to 3.58 ) \\
\hline Cancer, no LC & 6726 & 1031 & 15.3 & 1.00 & (reference) \\
\hline Cancer, LC & 6726 & 1154 & 17.2 & 1.15 & (1.05 to 1.27$)$ \\
\hline Respiratory disease, no LC & 4524 & 837 & 18.5 & 1.00 & (reference) \\
\hline Respiratory disease, LC & 4524 & 986 & 21.8 & 1.24 & (1.12 to 1.38$)$ \\
\hline Circulatory disease, no LC & 4385 & 384 & 8.8 & 1.00 & (reference) \\
\hline Circulatory disease, LC & 4385 & 530 & 12.1 & 1.47 & (1.28 to 1.70$)$ \\
\hline Infectious disease, no LC & 3518 & 807 & 22.9 & 1.00 & (reference) \\
\hline
\end{tabular}


Table 3 Stratified analysis and effects of cirrhosis-related clinical indicators on 30-day mortality of ICU patients (Continued)

\begin{tabular}{|c|c|c|c|c|c|}
\hline & \multirow[t]{2}{*}{$\mathrm{n}$} & \multicolumn{4}{|c|}{ 30-day mortality } \\
\hline & & Deaths & Mortality, \% & OR & $(95 \% \mathrm{Cl})^{\mathrm{a}}$ \\
\hline Infectious disease, LC & 3518 & 983 & 27.9 & 1.32 & (1.19 to 1.48$)$ \\
\hline Injury and poisoning, no LC & 2918 & 192 & 6.6 & 1.00 & (reference) \\
\hline Injury and poisoning, LC & 2918 & 345 & 11.8 & 1.95 & (1.61 to 2.35$)$ \\
\hline Symptom-defined conditions, no LC & 862 & 123 & 14.3 & 1.00 & (reference) \\
\hline Symptom-defined conditions, LC & 862 & 194 & 22.5 & 1.86 & (1.43 to 2.42$)$ \\
\hline Genitourinary disease, no LC & 762 & 38 & 5.0 & 1.00 & (reference) \\
\hline Genitourinary disease, LC & 762 & 59 & 7.7 & 1.74 & (1.10 to 2.75$)$ \\
\hline Endocrine disease, no LC & 605 & 24 & 4.0 & 1.00 & (reference) \\
\hline Endocrine disease, LC & 605 & 31 & 5.1 & 1.36 & (0.76 to 2.43 ) \\
\hline Musculoskeletal disease, no LC & 598 & 17 & 2.8 & 1.00 & (reference) \\
\hline Musculoskeletal disease, LC & 598 & 42 & 7.0 & 3.49 & (1.79 to 6.82$)$ \\
\hline Mental disorder, no LC & 278 & 0 & 0.0 & 1.00 & (reference) \\
\hline Mental disorder, LC & 278 & 0 & 0.0 & - & - \\
\hline Neurological disease, no LC & 304 & 11 & 3.6 & 1.00 & (reference) \\
\hline Neurological disease, LC & 304 & 25 & 8.2 & 2.94 & (1.30 to 6.65$)$ \\
\hline Skin disease, no LC & 291 & 7 & 2.4 & 1.00 & (reference) \\
\hline Skin disease, LC & 291 & 8 & 2.8 & 1.23 & (0.35 to 4.39$)$ \\
\hline Tumor, no LC & 254 & 2 & 0.8 & 1.00 & (reference) \\
\hline Tumor, LC & 254 & 5 & 2.0 & 3.33 & (0.51 to 21.8$)$ \\
\hline Blood disease, no LC & 76 & 5 & 6.6 & 1.00 & (reference) \\
\hline Blood disease, LC & 76 & 3 & 4.0 & 0.38 & (0.05 to 2.84$)$ \\
\hline Congenital anomalies, no LC & 40 & 1 & 2.5 & 1.00 & (reference) \\
\hline Congenital anomalies, LC & 40 & 0 & 0.0 & - & - \\
\hline Disease of perinatal period, no LC & 33 & 1 & 3.0 & 1.00 & (reference) \\
\hline Disease of perinatal period, LC & 33 & 0 & 0.0 & - & - \\
\hline Complications of pregnancy, no LC & 7 & 0 & 0.0 & 1.00 & (reference) \\
\hline Complications of pregnancy, LC & 7 & 1 & 14.3 & - & - \\
\hline 0 CCl score, no LC & 10,476 & 1066 & 10.2 & 1.00 & (reference) \\
\hline o CCl score, LC & 1165 & 101 & 8.7 & 0.97 & (0.77 to 1.23$)$ \\
\hline $1 \mathrm{CCl}$ score, no LC & 8899 & 731 & 8.2 & 1.00 & (reference) \\
\hline 1 CCl score, LC & 6656 & 818 & 12.3 & 1.38 & (1.21 to 1.58$)$ \\
\hline 2 CCl score, no LC & 6573 & 592 & 9.0 & 1.00 & (reference) \\
\hline 2 CCI score, LC & 5116 & 592 & 11.6 & 1.49 & (1.31 to 1.70$)$ \\
\hline 3 CCI score, no LC & 3897 & 355 & 9.1 & 1.00 & (reference) \\
\hline 3 CCl score, LC & 5478 & 760 & 13.9 & 1.45 & (1.25 to 1.69$)$ \\
\hline $4 \mathrm{CCl}$ score, no LC & 1823 & 209 & 11.5 & 1.00 & (reference) \\
\hline 4 CCl score, LC & 7085 & 1325 & 18.7 & 1.40 & (1.17 to 1.67 ) \\
\hline$\geq 5 \mathrm{CCl}$ score, no LC & 5529 & 1093 & 19.8 & 1.00 & (reference) \\
\hline$\geq 5$ CCl score, LC & 11,697 & 2299 & 19.7 & 1.08 & (0.99 to 1.18 ) \\
\hline
\end{tabular}

$\overline{A D S}$ alcohol dependence syndrome, $C l$ confidence interval, $G$ gastrointestinal, $H B V$ hepatitis $B$ virus, $H C V$ hepatitis $C$ virus, ICU intensive care unit, $L C$ liver cirrhosis, OR odds ratio

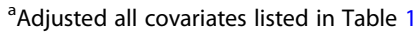


death in Taiwan [32], so it is reasonable that cirrhotic patients with liver cancer would have higher ICU mortality than those without it.

Regarding increased ICU mortality and medical expenditures, there are some possible explanations why patients with liver cirrhosis had worse outcomes. First, patients with liver cirrhosis are presumed to have impaired immune function and are thus more susceptible to severe infection, leading to higher mortality [33, 34]. A previous study demonstrated that infection increased mortality in patients with cirrhosis of liver fourfold, with $30 \%$ of patients dying within a month of infection and another $30 \%$ dying within a year [35]. This was consistent with our finding that septicemia and pneumonia in patients with cirrhosis were associated with higher ICU mortality. Second, portal hypertension and subsequent esophageal variceal bleeding and ascites play major roles as complications of cirrhosis and are associated with 1year mortality of nearly $20 \%[1,5,36]$. Third, as cirrhosis progresses, the development of renal vasoconstriction leads to hepatorenal syndrome. Renal failure is an indicator of end-stage liver disease and increases mortality risk by seven times, with half of patients dying within a month [37]. In our study, acute renal failure was also significantly associated with ICU mortality. To reduce ICU mortality in patients with liver cirrhosis, health care teams should optimize management of these specific issues according to updated guidelines.

Concerning long-term outcomes, variables such as serum albumin or bilirubin levels, ascites, encephalopathy, and prothrombin time for the Child-Pugh score are the most common independent predictors of mortality in patients with liver cirrhosis [5]. In our national cohort, ICU mortality among patients with liver cirrhosis increased with numbers of cirrhosis-related clinical conditions. These findings were compatible with previous studies demonstrating high mortality in cirrhotic patients with renal failure and gastrointestinal hemorrhage [37, 38]. However, our study focused on impacts on long-term mortality after ICU discharge, which was not investigated before. Considering specific management of these factors for ICU patients with cirrhosis of the liver is warranted to reduce mortality.

The present study has strengths of large sample sizes and adjustment for potential confounding factors by propensity score-matching method in a nationwide population-based retrospective cohort. It also has some limitations encountered in research based on secondary data. First, detailed information on laboratory data, physical examinations, and hemodynamic parameters was not available from reimbursement claim data. For example, the international normalized ratio of prothrombin time, bilirubin and creatinine level in blood would help to predict outcomes among cirrhosis patients [13,
14]. Second, severity of liver cirrhosis noted by ChildPugh score, Model For End-Stage Liver Disease score or other criteria was not found in reimbursement data for risk stratification of ICU mortality. Third, though the accuracy of major diagnosis codes from the Taiwan $\mathrm{Na}$ tional Health Insurance Research Database has been accepted by scientific journals [17-20], the validity of liver cirrhosis, comorbidity and complication codes employed in this study might still be questioned. To reduce the possibility of misdiagnosis or miscoding, we applied inclusive criteria of at least two visits for medical services with physician's primary diagnosis of liver cirrhosis. In addition, an important factor influencing ICU outcome is Do Not Resuscitate orders. However, we have no data regarding the Do Not Resuscitate order in this study because of the limitations of Taiwan's National Health Insurance Research Database. Finally, we could not exclude the possibility that some patients with hepatitis without cirrhosis were included in cirrhotic group in this study because the diagnosis error by physicians may occur in the clinical settings.

\section{Conclusions}

In conclusion, this nationwide population-based study showed that patients with liver cirrhosis admitted to ICU have higher ICU and one-year mortality after discharge in patterns that closely correlate with medical conditions and specific scenarios. These findings can help health care providers develop specific protocols to improve prognosis and long-term survival rates for ICU patients with liver cirrhosis.

\section{Supplementary information}

Supplementary information accompanies this paper at https://doi.org/10. 1186/s12876-020-1163-1.

Additional file 1: Figure S1. Flow chart for selecting intensive care unit patients with or without liver cirrhosis, 2006-2013. Table S1. International Classification of Diseases, Ninth Revision, Clinical Modification (ICD-9-CM) codes of diseases. Table S2. Stratified analysis and effects of cirrhosisrelated clinical indicators on ICU mortality. Table S3. Stratified analysis and effects of cirrhosis-related clinical indicators on one-year mortality of ICU patients. Table S4. The actual survival starting at the day of ICU admission in patients with and without liver cirrhosis

\section{Abbreviations \\ Cl: Confidence interval; ICD-9-CM: International Classification of Diseases, 9th Revision, Clinical Modification; ICU: Intensive care unit; OR: Odds ratio}

\section{Acknowledgements}

This study is based in part on data obtained from the National Health Insurance Research Database provided by the Bureau of National Health Insurance of the Taiwan Department of Health and managed by the National Health Research Institutes. The interpretation and conclusions contained herein do not represent those of the Bureau of National Health Insurance, the Taiwan Department of Health, or the National Health Research Institutes. 


\section{Authors' contributions}

Conception and design: YFH, CSL, YGC, CCY, RJC, TLC, CCL; Acquisition of data: TLC; Analysis of data: CCL; Interpretation of data: YFH, CSL, YGC, CCY, RJC, TLC, CCL; 2.Drafting the article: YFH, CCL; Revising it critically for important intellectual content: YFH, CSL, YGC, CCY, RJC, TLC, CCL; Final approval of the version to be published: YFH, CSL, YGC, CCY, RJC, TLC, CCL: Agreement to be accountable for all aspects of the work: YFH, CSL, YGC, $C C Y, R J C, T L C, C C L ;$ RJC contributed equally with the first author; TLC contributed equally with the corresponding author. All authors read and approved the final manuscript.

\section{Funding}

Taiwan's Ministry of Science and Technology (MOST108-2221-E-038-006; MOST106-2314-B-038-036-MY3). The funders had no role in the design, data collection, data analysis, data interpretation or writing of this paper, nor in the decision to submit the paper for publication.

\section{Availability of data and materials}

Our data will not be shared because of the regulations from the Health and Welfare Data Science Center (HWDC). The data underlying this study is from the National Health Insurance Research Database (NHIRD), which has been transferred to the HWDC. Interested researchers can obtain the data through formal application to the HWDC, Department of Statistics, Ministry of Health and Welfare, Taiwan (http://dep.mohw.gov.tw/DOS/np-2497-113.html).

\section{Ethics approval and consent to participate}

This study was conducted in accordance with the Helsinki Declaration. To protect personal privacy, the electronic database was decoded with patient identifications scrambled for further academic access for research. According to Taiwan National Health Research Institutes regulations, informed consent is not required because patient identifications were decoded and scrambled. Ethical approval for this study (TMU-JIRB-201504008) was provided by the Institutional Review Board of Taipei Medical University.

\section{Consent for publication}

Not applicable.

\section{Competing interests}

The authors declare that they have no competing interests.

\section{Author details}

'Department of Anesthesiology, Taitung MacKay Memorial Hospital, Taitung, Taiwan. ${ }^{2}$ Department of Anesthesiology, Shuang Ho Hospital, Taipei Medical University, New Taipei City, Taiwan. ${ }^{3}$ Department of Anesthesiology, School of Medicine, College of Medicine, Taipei Medical University, Taipei, Taiwan. ${ }^{4}$ Department of Anesthesiology, Taipei Medical University Hospital, Taipei, Taiwan. ${ }^{5}$ Anesthesiology and Health Policy Research Center, Taipei Medical University Hospital, Taipei, Taiwan. ${ }^{6}$ Department of Surgery, China Medical University Hospital, Taichung, Taiwan. 'Department of Surgery, University of Illinois, Chicago, USA. ${ }^{8}$ Department of Surgery, Taipei Medical University Hospital, Taipei, Taiwan. ${ }^{9}$ Department of Surgery, School of Medicine, College of Medicine, Taipei Medical University, Taipei, Taiwan. ${ }^{10}$ Department of Anesthesiology, Wan Fang Hospital, Taipei Medical University, Taipei, Taiwan. ${ }^{11}$ Research Center of Big Data and Meta-Analysis, Wan Fang Hospital, Taipei Medical University, Taipei, Taiwan. ${ }^{12}$ School of Chinese Medicine, College of Chinese Medicine, China Medical University, Taichung, Taiwan.

\section{Received: 3 June 2019 Accepted: 6 January 2020}

\section{Published online: 16 January 2020}

\section{References}

1. Tsochatzis EA, Bosch J, Burroughs AK. Liver cirrhosis. Lancet. 2014;383:1749-61.

2. Lozano R, Naghavi M, Foreman K, et al. Global and regional mortality from 235 causes of death for 20 age groups in 1990 and 2010: a systematic analysis for the global burden of disease study 2010. Lancet. 2012;380:2095-128.

3. Blachier M, Leleu H, Peck-Radosavljevic M, Valla DC, Roudot-Thoraval F. The burden of liver disease in Europe: a review of available epidemiological data. J Hepatol. 2013;58:593-608.

4. Olson JC, Wendon JA, Kramer DJ, et al. Intensive care of the patient with cirrhosis. Hepatology. 2011;54:1864-72.
5. D'Amico G, Garcia-Tsao G, Pagliaro L. Natural history and prognostic indicators of survival in cirrhosis: a systematic review of 118 studies. J Hepatol. 2006;44:217-31.

6. Galbois A, Trompette ML, Das V, et al. Improvement in the prognosis of cirrhotic patients admitted to an intensive care unit: a retrospective study. Eur J Gastroenterol Hepatol. 2012;24:897-904.

7. Thomson SJ, Moran C, Cowan ML, et al. Outcomes of critically ill patients with cirrhosis admitted to intensive care: an important perspective from the non-transplant setting. Aliment Pharmacol Ther. 2010;32:233-43.

8. Cholongitas E, Senzolo M, Patch D, Shaw S, Hui C, Burroughs AK. Review article: scoring systems for assessing prognosis in critically ill adult cirrhotics. Aliment Pharmacol Ther. 2006;24:453-64.

9. Das V, Boelle PY, Galbois A, et al. Cirrhotic patients in the medical intensive care unit: early prognosis and long-term survival. Crit Care Med. 2010;38:2108-16.

10. Levesque E, Hoti E, Azoulay D, et al. Prospective evaluation of the prognostic scores for cirrhotic patients admitted to an intensive care unit. J Hepatol. 2012;56:95-102.

11. Theocharidou E, Pieri G, Mohammad AO, et al. The Royal Free Hospital score: a calibrated prognostic model for patients with cirrhosis admitted to intensive care unit. Comparison with current models and CLIF-SOFA score. Am J Gastroenterol. 2014;109:554-62.

12. Emerson P, McPeake J, O'Neill A, et al. The utility of scoring systems in critically ill cirrhotic patients admitted to a general intensive care unit. J Crit Care. 2014;29:1131.

13. Pugh RN, Murray-Lyon IM, Dawson JL, Pietroni MC, Williams R. Transection of the oesophagus for bleeding oesophageal varices. Br J Surg. 1973;60:646-9.

14. Malinchoc M, Kamath PS, Gordon FD, Peine CJ, Rank J, ter Borg PC. A model to predict poor survival in patients undergoing transjugular intrahepatic portosystemic shunts. Hepatology. 2000;31:864-71.

15. Knaus WA, Draper EA, Wagner DP, Zimmerman JE. APACHE II: a severity of disease classification system. Crit Care Med. 1985;13:818-29.

16. Vincent JL, Moreno R, Takala J, et al. SOFA (Sepsis-related organ failure assessment) score to describe organ dysfunction/failure: working group on Sepsis-related problems of the European Society of Intensive Care Medicine. Intensive Care Med. 1996;22:707-10.

17. Cheng CL, Kao YH, Lin SJ, Lee CH, Lai ML. Validation of the National Health Insurance Research Database with ischemic stroke cases in Taiwan. Pharmacoepidemiol Drug Saf. 2011:20:236-42.

18. Lin CS, Lin SY, Chang CC, Wang HH, Liao CC, Chen TL. Postoperative adverse outcomes after non-hepatic surgery in patients with liver cirrhosis. Br J Surg. 2013;100:1784-90.

19. Yeh CC, Liao CC, Chang YC, et al. Adverse outcomes after noncardiac surgery in patients with diabetes: a nationwide population-based retrospective cohort study. Diabetes Care. 2013;36:3216-21.

20. Lin JA, Liao CC, Lee YJ, Wu CH, Huang WQ, Chen TL. Adverse outcomes after major surgery in patients with systemic lupus erythematosus: a nationwide population-based study. Ann Rheum Dis. 2014;73:1646-51.

21. Wehler M, Kokoska J, Reulbach U, Hahn EG, Strauss R. Short-term prognosis in critically ill patients with cirrhosis assessed by prognostic scoring systems. Hepatology. 2001;34:255-61.

22. Gildea TR, Cook WC, Nelson DR, et al. Predictors of long-term mortality in patients with cirrhosis of the liver admitted to a medical ICU. Chest. 2004; 126:1598-603

23. Cholongitas E, Calvaruso V, Senzolo M, et al. RIFLE classification as predictive factor of mortality in patients with cirrhosis admitted to intensive care unit. J Gastroenterol Hepatol. 2009;24:1639-47.

24. Juneja D, Gopal PB, Kapoor D, Raya R, Sathyanarayanan M, Malhotra P. Outcome of patients with liver cirrhosis admitted to a specialty liver intensive care unit in India. J Crit Care. 2009;24:387-93.

25. Freire $P$, Romaozinho JM, Amaro P, Ferreira M, Sofia C. Prognostic scores in cirrhotic patients admitted to a gastroenterology intensive care unit. Rev Esp Enferm Dig. 2011;103:177-83.

26. Bahirwani R, Ghabril M, Forde KA, et al. Factors that predict short-term intensive care unit mortality in patients with cirrhosis. Clin Gastroenterol Hepatol. 2013;11:1194-200.

27. Dombrovskiy VY, Martin AA, Sunderram J, Paz HL. Rapid increase in hospitalization and mortality rates for severe sepsis in the United States: a trend analysis from 1993 to 2003. Crit Care Med. 2007;35:1244-50.

28. Blot $\mathrm{S}$, Koulenti D, Dimopoulos $\mathrm{G}$, et al. Prevalence, risk factors and mortality for ventilator-associated pneumonia in middle-aged, old and very old critically ill patients. Crit Care Med. 2014;42:601-9. 
29. Ai-Ping C, Lee KH, Lim TK. In-hospital and 5-year mortality of patients treated in the ICU for acute exacerbation of COPD: a retrospective study. Chest. 2005;128:518-24.

30. De Franchis R. Evolving consensus in portal hypertension: report of the Baveno IV consensus workshop on methodology of diagnosis and therapy in portal hypertension. J Hepatol. 2005;43:167-76.

31. D'Amico G, Morabito A, Pagliaro L, Marubini E. Survival and prognostic indicators in compensated and decompensated cirrhosis. Dig Dis Sci. 1986; 31:468-75.

32. Kee KM, Lu SN. Hospital- and community-based screenings for hepatocellular carcinoma in Taiwan. Oncology. 2011;81:36-40.

33. Christou L, Pappas G, Falagas ME. Bacterial infection-related morbidity and mortality in cirrhosis. Am J Gastroenterol. 2007;102:1510-7.

34. Thulstrup AM, Sørensen HT, Schønheyder HC, Møller JK, Tage-Jensen U. Population-based study of the risk and short-term prognosis for bacteremia in patients with liver cirrhosis. Clin Infect Dis. 2000;31:1357-61.

35. Arvaniti V, D'Amico G, Fede G, et al. Infections in patients with cirrhosis increase mortality four-fold and should be used in determining prognosis. Gastroenterology. 2010;139:1246-56.

36. Runyon BA. Management of adult patients with ascites due to cirrhosis: an update. Hepatology. 2009:49:2087-107.

37. Fede G, D'Amico G, Arvaniti V, et al. Renal failure and cirrhosis: a systematic review of mortality and prognosis. J Hepatol. 2012;56:810-8.

38. Nidegger $\mathrm{D}$, Ragot $\mathrm{S}$, Berthelemy $\mathrm{P}$, et al. Cirrhosis and bleeding: the need for very early management. J Hepatol. 2003;39:509-14.

\section{Publisher's Note}

Springer Nature remains neutral with regard to jurisdictional claims in published maps and institutional affiliations.

Ready to submit your research? Choose BMC and benefit from:

- fast, convenient online submission

- thorough peer review by experienced researchers in your field

- rapid publication on acceptance

- support for research data, including large and complex data types

- gold Open Access which fosters wider collaboration and increased citations

- maximum visibility for your research: over $100 \mathrm{M}$ website views per year

At BMC, research is always in progress.

Learn more biomedcentral.com/submissions 